\title{
Percepciones comunitarias relativas a la prevención del dengue en asentamientos humanos afectados. Lima-Perú, 2015
}

Gloria Carmona(a)

Luis Fernando Donaires(b)

Carmona G, Donaires LF. Community perceptions about dengue prevention in human settlements. Lima-Perú, 2015. Interface (Botucatu). 2016; 20(59):839-52.

This is a qualitative study, aimed at describing community perceptions on dengue prevention in affected areas of Lima. A series of interviews were conducted in six of those areas using the health belief model approach. Respondents noted several factors about sanitation, behaviors and information that could increase their exposure to dengue. They expressed that this illness would affect their lives in different ways, in aspects related to work, family and economy. They recognized their share of responsibility on prevention, but also talked about the importance of health promoters work, media and community leaders. Among the preventive measures they mentioned, there were a few with no evidence of effectiveness. The main identified problems were lack of health professionals, community participation and preventive activities. They also noted that most activities were discontinued after outbreaks. The findings provide an opportunity to discuss and reflect on the preventive work done to face dengue.

Keywords: Dengue. Human settlements. Social perception. Intersectoral action.
Con el objetivo de describir las percepciones comunitarias para la prevención del dengue en las localidades afectadas de Lima, realizamos un estudio cualitativo a través de entrevistas desde el modelo de creencias en salud. Los entrevistados mencionaron diversos factores de saneamiento, conductas e información que incrementan su exposición al dengue, expresando que los afectaría en aspectos laborales, familiares y económicos, asumiendo parte de la responsabilidad en la prevención y recalcando la labor de los promotores de salud, medios de comunicación y líderes comunitarios. Entre las medidas preventivas mencionadas, se incluyeron medidas sin evidencia de efectividad. Las principales barreras expresadas fueron las siguientes, caraterizadas como insuficientes: cantidad de profesionales de salud, participación comunitaria, organización en las actividades preventivas y discontinuidad luego de los brotes. La discusión y reflexión sobre estos contextos promueve una oportunidad en el abordaje de la labor preventiva hacia el dengue.

Palabras clave: Dengue. Percepción social. Acción intersectorial. Asentamientos humanos. (a) Instituto Nacional
de Salud, Centro
Nacional de Salud
Pública, Unidad de
análisis y generación
de evidencias en salud
Pública (UNAGESP).
Cápac Yupanqui
1400, Jesus María,
Lima 11. Lima, Perú.
gcarmona@ins.gob.pe
(b) Instituto Nacional de
Salud, Centro Nacional
de Salud Pública. Lima,
Perú. fernandod1225@
gmail.com 


\section{Introduccion}

En la actualidad el dengue es la enfermedad viral transmitida por vectores más importante a nivel mundial en términos de morbilidad e impacto económico1. La Organización Mundial de la Salud ha estimado que 50-100 millones de infecciones ocurren anualmente y que más de 2,5 billones de personas se encuentran en riesgo ${ }^{2}$. En América Latina se ha observado un incremento de los casos en los últimos años, proviniendo el $21 \%$ de países andinos ${ }^{3}$. En el Perú el Dengue se ha extendido desde la Amazonía a la costa norte y a la capital. El primer brote notificado en Lima fue el año 2005, confirmándose en ese año hasta $146 \operatorname{casos}^{4-6}$. En Lima, se han registrado numerosos brotes en diversos asentamientos humanos $(\mathrm{AAHH})$, los cuales son las comunidades urbanas más carentes y vulnerables ${ }^{4,7-9}$. Uno de los brotes de mayor magnitud ocurrió en el sur de Lima durante el 2014, con un total de 99 casos confirmados ${ }^{10}$.

El Plan nacional Multisectorial e Intergubernamental del Prevención y control de dengue en el Perú, incluye entre las áreas priorizadas a siete distritos de Lima, las cuales presentan la mayor densidad poblacional. Sus objetivos incluyen: estrategias de comunicación masiva; sensibilización intersectorial, fortaleciendo redes organizacionales; el fortalecimiento de los sistemas de vigilancia y monitoreo, el desarrollo de entornos saludables a fin de reducir los índices de infestación a través de diversas actividades; como en el recojo oportuno y adecuado de residuos sólidos e inservibles, fumigaciones y promoviendo la educación sanitaria ${ }^{11}$. Sin embargo, la efectividad de las estas intervenciones, depende de su continuidad, cobertura, y principalmente de la ejecución de conductas preventivas continuas por parte de la comunidad ${ }^{12}$. Esta ejecución está influenciada tanto de las percepciones individuales y colectivas sobre el riesgo a la enfermedad, como del reconocimiento de la vulnerabilidad según las condiciones de saneamiento en las que las personas viven. El conjunto de estas interpretaciones socio-ecológicas está asociada con la posibilidad de tomar la decisión de ejecutar alguna medida preventiva ${ }^{13,14}$.

Otras barreras identificadas en las actividades preventivas son la discontinuidad de las actividades una vez superado el brote que las origina y la escasa participación comunitaria que dificulta estrategias articuladas e intersectoriales en el control del vector ${ }^{15-17}$. La actual evidencia científica ha demostrado que aun en condiciones óptimas de control del vector, la transmisión puede restablecerse si no se sostiene la participación integral de los diversos actores sociales implicados, desde los tomadores de decisiones hasta las comunidades afectadas, a fin de asegurar la prevención y control de la enfermedad ${ }^{18}$.

Considerando la intensa movilización de personal de salud y de recursos para el control de brotes, indagar y documentar las percepciones en relación a la prevención y control del dengue desde la óptica de los actores implicados, podría contribuir en la optimización de las actividades preventivopromocionales a través de la participación comunitaria integral, por lo cual el presente estudio tuvo como objetivo conocer las percepciones de diversos actores comunitarios en relación a la prevención del dengue en diversos AAHH afectados de Lima, Perú.

\section{Material y método}

\section{Diseño de estudio}

Realizamos un estudio cualitativo que utiliza un enfoque ecológico que entiende a la salud como el resultado de un complejo grupo de interacciones entre factores ecológicos, biológicos y socioculturales, empleándose el marco teórico del modelo de creencias en salud ${ }^{19}$ (ver Diagrama 1). Así, las percepciones en relación a la prevención del dengue y sus determinantes tuvieron lugar dentro de particulares circunstancias entre los actores sociales como características sanitarias, socioeconómicas, de información, u otras; y no solamente como resultado de los factores preventivos sanitarios ${ }^{20-22}$. 


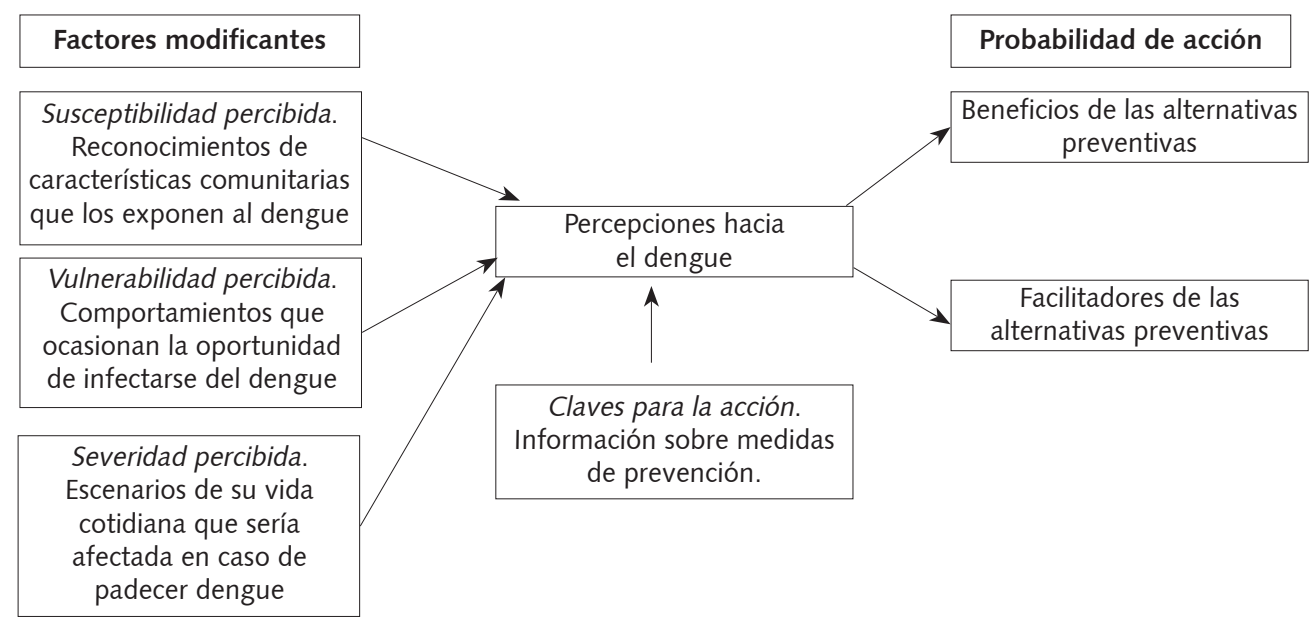

Diagrama 1. Referente conceptual para la ejecución de prácticas preventivas.

Fuente: Adaptación al modelo de Creencias en Salud propuesto por Hochbaum et al..$^{23}$

\section{Descripción de la población de estudio}

Los AAHH participantes de este estudio son comunidades urbano marginales ubicadas en las faldas medias o superiores de los cerros limeños, constituidas principalmente por poblaciones inmigrantes procedentes de la sierra y selva del país. Estos $\mathrm{AAHH}$ en general sufren de persistentes deficiencias en el acceso al agua, saneamiento ambiental y transporte. El hacinamiento, la localización inadecuada y la infraestructura precaria de sus viviendas; las dificultades bilaterales de acceso a diversos servicios, como de salud y de recolección de desechos por la ausencia de rutas asfaltadas obstaculizan el acceso a las personas y a los medios de transporte. Estos factores contribuyen a riesgos de contaminación y accidentes, representando una manifestación de desigualdad ${ }^{24}$. Por otro lado, el clima de estos AAHH presenta un alto nivel de humedad atmosférica produciendo neblina persistente, las lluvias afecta especialmente a estos cerros ocasionando desde acumulación de agua en sus techos precarios hasta huaycos.

\section{Estrategias de selección de casos y muestreo}

Se seleccionaron por conveniencia seis AAHH de Lima Metropolitana, con el antecedente de haber presentado los mayores incidencias de casos notificados de dengue en los últimos tres años según los reportes oficiales de las diferentes unidades de epidemiología ${ }^{8,9}$. Al interior de cada AAHH se trabajó con un muestreo por cuotas intentando representar al enfoque de ecosalud ${ }^{25}$; en el que se incluyó a colaboradores y personal de salud local del programa de control de enfermedades Metaxénicas, líderes comunitarios identificados durante las visitas domiciliarias, según opiniones de la población, y residentes hasta alcanzar la saturación de información. Manteniendo el enfoque de conveniencia se dio prioridad de participación voluntaria a las viviendas con menor acceso a servicios básicos de saneamiento. Todos los participantes habían vivido o trabajado en la zona por un periodo mayor de tres años. 


\section{Técnicas de recolección de datos}

Realizamos entrevistas grabadas, semiestructuradas, abiertas e indirectas, a fin de evitar la limitación de categorías predeterminadas así como la impresión de confrontación al participante. El proceso de elaboración de la guía semiestructurada fue elaborado en referencia al modelo de creencias en salud además del marco teórico del tema propuesto y adaptado según el contexto de cada comunidad y participante. Entrevistamos a pobladores, líderes comunitarios y personal de salud hasta lograr la saturación de información. Las entrevistas fueron realizadas por el investigador principal en las viviendas o centros de salud de los participantes, con una duración de treinta minutos como promedio, luego de cada entrevista fueron elaborados las notas de campo y memos analíticos necesarios.

\section{Técnica de análisis de datos}

Las entrevistas y las anotaciones adicionales fueron transcritas en un procesador de textos y codificadas a través del software para análisis cualitativo ATLAS. Ti (versión de prueba). El análisis se dividió en las siguientes fases: (1) lectura de las entrevistas y elección de códigos, (2) nueva lectura de entrevistas y codificación, (3) análisis por códigos, diferenciando cada entrevista según actor social para encontrar regularidades y diferencias, y (4) elaboración de matrices de concentración de datos según códigos a partir de la información encontrada en las entrevistas, permitiendo focalizar resultados y establecer una relación con la teoría existente. Este análisis incluyó el proceso dinámico de discusión e interpretación de los hallazgos con el equipo de investigación, lo que permitió identificar relaciones y diferencias entre las categorías de códigos y los participantes del estudio según tipo de actor social. En los criterios de diseño, descripción y análisis se ha considerado el COREQ Checklist ${ }^{26}$.

\section{Aspectos éticos}

El presente estudio fue aprobado por el comité de ética del Instituto Nacional de Salud (INS). Se obtuvo el consentimiento de las autoridades locales para acceder a los AAHH seleccionados.

\section{Resultados}

Se entrevistaron a 34 participantes entre residentes, líderes comunitarios (presidentes de asociaciones vecinales, comedores populares o párrocos) y personal de salud (enfermeras, médicos o promotores de salud) miembros de la estrategia de prevención de enfermedades metaxénicas y zoonosis de los AAHH seleccionados. Se realizaron además 12 conversaciones informales. Sus percepciones en relación a la prevención del dengue han sido organizadas en dos enfoques: El primer enfoque, se refiere a la interpretación de factores modificantes, que incluyen tanto características socioeconómicas, demográficas y/o estructurales, como la información recibida sobre el dengue a través de campañas educativas, consejos o experiencias recibidas por sus pares. Esta interpretación, se refleja en la susceptibilidad, vulnerabilidad y severidad percibida (ver diagramas 2 y 3 ). El segundo enfoque, está conformado por las barreras o facilitadores percibidas hacia la probabilidad de ejecutar alguna alternativa preventiva.

En relación a los factores modificantes los participantes manifestaron diversas características comunitarias que los hacen susceptibles y vulnerables al dengue al, como el acceso restringido al servicio de agua potable, el cual se presenta por horas, que favorece el almacenamiento de agua en tachos o tanques. También mencionaron la acumulación de agua en techos o inservibles durante las lluvias.

Además, comentaron que la eliminación de aguas residuales en las calles por parte de algunos vecinos y las dificultades tanto en la recolección de basura como en su embalaje representa factores de riesgo para el dengue. 


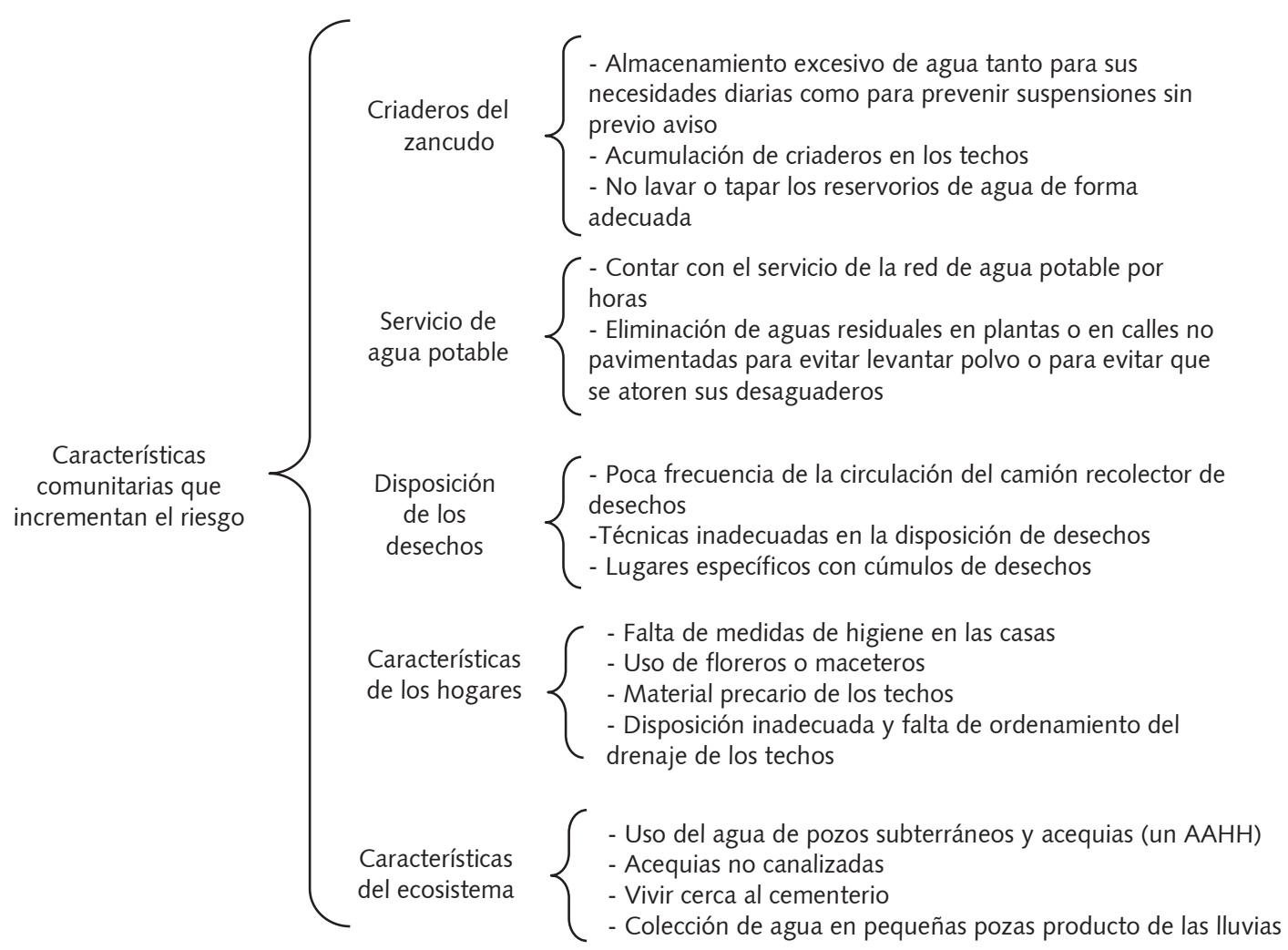

Diagrama 2. Percepciones en torno a la susceptibilidad y vulnerabilidad hacia el dengue

Fuente: Adaptación al modelo de Creencias en Salud propuesto por Hochbaum et al. ${ }^{23}$

Residentes y líderes
comunitarios $\left\{\begin{array}{l}\text { - Puede ocasionar la muerte } \\ \text { - Incapacidad temporal para el trabajo } \\ \text { - Ocasionaría "descuidar a los hijos, familia y hogar" } \\ \text { - Tener que gastar dinero adicional } \\ \text { - Posibilidad de contagiar a familiares } \\ \text { - Afecta más a niños }\end{array}\right.$

Diagrama 3. Percepciones en torno a la severidad percibida 
En relación a la aplicación de pautas de prevención del dengue, los entrevistados reconocieron un descuido en el lavado y tapado adecuado de los reservorios de agua, ya sea por cansancio o desinterés del poblador, que prioriza otros problemas.

En un AA.HH., el personal de salud reconoció que los pobladores aún hacen uso del agua de algunas acequias o pozos subterráneos.

“Tenemos 2 problemas básicos; ;.. los pozos subterráneos y las acequias. Las acequias son aguas superficial, y esa agua que sale de todas partes se junta en una sequía, como tipo desagüe....

Ellos cavan 2 metros y empieza a salir el agua, y usan esa agua para lavar, y algunos donde no hay red, usan para tomar. Y como el 2012 como tuvimos dengue, se les obligó que clausuren ese pozo, pero igual siguen usando". (Enfermera)

En todos los AA.HH el personal de salud manifestó que existe resistencia en algunos pobladores a las visitas domiciliarias y que en varios domicilios constataban prácticas inadecuadas que incrementan la vulnerabilidad de dengue.

“... es que nuestras pacientes no toman las prevenciones del caso, pero con todo esto que hubo se ha estado visitando casa por casa, educando, diciéndoles que tienen que eliminar los reservorios, pero se rehúsan pues, no quieren que entres, que si lo he eliminado te dicen, pero si entras a sus casas ahí lo tienen por más que les explicas ahí siguen". (Enfermera)

Al indagar sobre las percepciones hacia la enfermedad diversos residentes y líderes comunitarios, describieron que los huevos del zancudo se crían y salen del agua almacenada en depósitos, floreros o plantas, y que los focos de crianza se ubican en las esquinas de las habitaciones, baños y maceteros. Los demás entrevistados agregaron que el mosquito procede de las plantas, árboles, parques o de las aguas sucias.

"He escuchado que es un zancudo que aparece del agua que está depositada en el agua sucia en los tachos, en las esquinas de las paredes y se te enroncha y duele cabeza y se contagia a la familia". (Residente)

Algunos participantes reconocieron no prestar atención a las propagandas informativas sobre el dengue transmitido a través de los medios de comunicación. Varios líderes comunitarios expresaron que a pesar de haber participado en charlas informativas no comprendían porqué el zancudo es peligroso si anteriormente ya les había picado sin mayores consecuencias. Solo el personal y colaboradores de salud mencionaron el nombre del vector causante de dengue, adicionando que en varias ocasiones algunos residentes solicitan aplicarse la vacuna preventiva contra el dengue y la falta de percepción del riesgo en la mayoría de vecinos. En contraste a ello, la mayoría de los residentes y líderes entrevistados, se reconoció vulnerable para la infección por dengue. Aquellos entrevistados que no se consideraron vulnerables plantearon como argumentos de su respuesta; el no vivir en la parte más alta del cerro, el evitar tener agua empozada, el estar informados de los casos anteriores y porque consideran al lavado de manos como conducta de prevención frente al dengue.

“Uno no está libre, puede ser que algunos tengan más riesgo, pero nos puede atacar por lo mismo que nos descuidamos, a veces el agua empozada se queda ahí, la basura se amontona en la parte de arriba y nacen los zancudos". (Residente)

“Debemos estar lavando bien nuestros tachos, no permitiendo que haya agua sucia, no formar aguas, limpiar nuestras casas, lavándonos bien las manos, yo creo que por no hacer eso les dado esa vez". (Residente) 
En relación a la severidad percibida, los entrevistados indicaron que de padecerla se verían afectados, desde un punto de vista laboral y económico (Diagrama 3). Señalaron además la posibilidad de contagio y muerte a causa del dengue, mencionando algunos síntomas.

Considerando que las claves para la acción están conformadas por la información adecuada de medidas preventivas frente al dengue, los entrevistados mencionaron diversas actividades; como el lavado y tapado de los depósitos de manera correcta, la fumigación, el uso de larvicidas, y la prohibición de llevar flores con agua a los cementerios (Tabla 1).

Las principales barreras identificadas en los residentes y líderes comunitarios incluyeron medidas preventivas sin evidencia de efectividad en la prevención del dengue, como el lavado de manos, el evitar vivir en el cerro, el evitar eliminar aguas residuales en las vías no asfaltadas, evitar acumular agua sucia, evitar que los niños tomen el agua almacenada en reservorios y quemar la ropa de modo que el humo generado elimine al vector, en la Tabla 1 se interpretan cuáles de estas medidas preventivas mencionadas son adecuadas o inadecuadas de acuerdo a la literatura científica revisada.

“...quemo ropa vieja y agarro un palito y prendo candela y así lo mato al zancudo porque así señorita entra el humo y se va". (Residente)

Tabla 1. Alternativas de prevención del dengue mencionadas según cada actor social

\begin{tabular}{lll}
\hline \multicolumn{1}{c}{ Residentes } & \multicolumn{1}{c}{ Líderes comunitarios } & \multicolumn{1}{c}{ Salud } \\
\hline Adecuadas & - Usar, tapar y lavar adecuada & - Usar, tapar y lavar adecuada y \\
- Contar con agua permanente de la red pública & y frecuentemente los reservorios. & frecuentemente los reservorios. \\
- Usar, tapar y lavar adecuada y frecuentemente los & - Higiene de los baños de las & - Eliminación de inservibles \\
reservorios de agua & viviendas. & - Control de criaderos a través de \\
- Fumigaciones & & visitas domiciliarias. \\
- Evitar almacenar agua por largos periodos & - Revisar la presencia de los & \\
- Remplazar agua por arena en maceteros & criaderos en los techos. & \\
- Uso de motitas (Abate) en el agua almacenada & & \\
Inadecuadas & & \\
- Vacuna contra el dengue & & \\
- Lavado de manos & & \\
- Aseo diario (bañarse) & & \\
- Quemando ropa ya que el humo aleja al mosquito & \\
- Evitar tomar agua empozada & \\
- Tener más limpieza en casa & \\
\hline
\end{tabular}

La barrera más reiterativa expresada por todos los entrevistados fue la falta de profesionales de salud en las actividades preventivas, siendo estas realizadas principalmente por promotores de salud.

“Las promotoras son nuestro brazo derecho, para salir nosotros tendríamos que cerrar [...] cuando paso el dengue cerramos, solamente atendíamos emergencia [...] es un abuso, van a 20 casas por turno, y le daban creo que 15 soles, aparte tienen que pagar su moto para que se trasladen". (Enfermera)

"Las promotoras han venido y han entrado a la casa, para ver si hay algo que pueda ocasionar el dengue, botellas con agua, esas cosas. Pero no todos los vecinos quieren porque tiene miedo a la municipalidad". (Residente)

Los líderes comunitarios entrevistados expresaron que el aviso sin anticipación sobre la distribución y los cortes de agua, ocasionan un almacenamiento de agua inadecuado. Fue referido también; que las actividades preventivas pocas veces llegan a las zonas más alejadas de los $\mathrm{AAHH}$, la necesidad de una 
mayor interacción con los centros de salud y la necesidad de que organicen sus actividades de visitas domiciliarias o charlas con anticipación, así como realizarlas antes del verano, que es cuando más casos de dengue ocurren. Además, indicaron que la negativa o resistencia de la comunidad a las visitas domiciliarias, se debía al temor o desconfianza de que el personal de salud notificaría al municipio aspectos de saneamiento o construcciones no registradas, lo cual conllevaría a multas y clausuras (Tabla 2).

“Hemos recibido la agresión verbal de la población, las personas que no nos dejaban entrar y veníamos al otro día y nos decían no tienen nada que hacer váyanse!... Ya que se podía hacer...". (Promotora de salud)

“Cuando acá vinieron a fumigar, entraron y cerraron las puertas por 2 horas.....Por lo menos fumigar ayuda un poco..., aunque hay gente que no deja que entren porque dicen que van a entrar a robar o a avisar a la municipalidad de los cuartos de la casa". (Residente)

Tabla 2. Percepciones sobre cuestiones relacionadas a las actividades de prevención del dengue

\begin{tabular}{|c|c|c|c|}
\hline & Residentes & Lideres & Salud \\
\hline $\begin{array}{l}\text { Claves para } \\
\text { la acción }\end{array}$ & $\begin{array}{l}\text {-Mensajes en Tv } \\
\text {-Mensajes en periódicos } \\
\text {-Fumigaciones en tiempos de } \\
\text { brote } \\
\text {-Visitas domiciliarias y charlas } \\
\text { por parte del personal de salud }\end{array}$ & $\begin{array}{l}\text {-Fumigaciones en casas y } \\
\text { parques } \\
\text {-Entrega de motitas (abate) } \\
\text { en las visitas domiciliarias } \\
\text {-Charlas preventivas por el } \\
\text { personal de salud }\end{array}$ & $\begin{array}{l}\text {-Trabajo preventivo promocional } \\
\text { multidisciplinario e intersectorial (colegios } \\
\text { comisarias, casas) en periodos de brotes }\end{array}$ \\
\hline Barreras & $\begin{array}{l}\text {-Se realizan más en verano } \\
\text {-Restricción en el acceso del } \\
\text { personal de salud a las casas por } \\
\text { no avisar o temor que revisen } \\
\text { sus viviendas } \\
\text {-Pocas charlas de prevención } \\
\text { en las zonas más alejadas de los } \\
\text { AAHH } \\
\text {-Escasa comunicación sobre } \\
\text { cuándo van a fumigar } \\
\text {-Falta de participación } \\
\text { de la comunidad en las } \\
\text { charlas organizadas por los } \\
\text { establecimientos de salud } \\
\text {-Ausencia de reuniones vecinales } \\
\text { para ver esos asuntos }\end{array}$ & $\begin{array}{l}\text { - Charlas por el párroco } \\
\text {-Poca comprensión de } \\
\text { algunos adultos y niños a las } \\
\text { diversas charlas informativas } \\
\text { recibidas sobre dengue } \\
\text { - Falta de aviso anticipado de } \\
\text { las suspensiones temporales } \\
\text { del servicio de agua } \\
\text {-Resistencia de la comunidad } \\
\text { a las visitas domiciliarias } \\
\text { por temor a notificaciones } \\
\text { municipales de las } \\
\text { instalaciones no registradas }\end{array}$ & $\begin{array}{l}\text { - Incentivo económico insuficiente a las } \\
\text { promotoras, quienes realizan la mayor carga } \\
\text { de estas actividades. } \\
\text {-Personal profesional y técnico de la salud } \\
\text { insuficiente para realizar las actividades } \\
\text { extramurales, ya que solo cuentan con un } \\
\text { equipo básico } \\
\text {-Actividades de prevención no sostenidas en } \\
\text { los periodos interbrote } \\
\text { - Organización deficiente durante las } \\
\text { actividades de fumigación en relación a la falta } \\
\text { de aviso de las fechas, horarios y duración } \\
\text {-Resistencia de la comunidad a las visitas } \\
\text { domiciliarias por no desear ser inspeccionados } \\
\text { ni desear eliminar los inservibles encontrados } \\
\text {-Resistencia al remplazo del agua por arena } \\
\text { en los floreros ubicados en las casas y en los } \\
\text { cementerios } \\
\text {-Recursos económicos insuficientes para el } \\
\text { transporte en las actividades de campo }\end{array}$ \\
\hline $\begin{array}{l}\text { Invitaría a } \\
\text { participar } \\
\text { en estas } \\
\text { actividades }\end{array}$ & $\begin{array}{l}\text {-Municipalidad } \\
\text {-Comité municipal } \\
\text {-Al párroco }\end{array}$ & $\begin{array}{l}\text {-Personal de limpieza y } \\
\text { saneamiento }\end{array}$ & $\begin{array}{l}\text {-Gobierno central (spots publicitarios, } \\
\text { propagandas, contrata de mayor personal) } \\
\text {-Comités comunitarios } \\
\text {-Parroquias }\end{array}$ \\
\hline $\begin{array}{l}\text { Responsable } \\
\text { de estas } \\
\text { actividades }\end{array}$ & $\begin{array}{l}\text {-Nosotros } \\
\text {-Todos } \\
\text {-Gobierno } \\
\text {-Posta médica }\end{array}$ & -Personal de salud & $\begin{array}{l}\text {-Saneamiento ambiental } \\
\text {-Personal de salud } \\
\text {-Comunidad }\end{array}$ \\
\hline
\end{tabular}


El personal de salud manifestó como barreras en las actividades preventivo-promocionales, su discontinuidad luego de los brotes, la falta de organización con anticipación al verano, a fin de contar con suficientes recursos humanos como promotoras de salud e insumos a distribuir en la comunidad (como depósitos, larvicidas y materiales informativos) y la escaza participación del municipio.

"Con la fumigación no se dio la orientación como: Señora no vuelva dentro de dos horas a su casa $[. .$.$] y hubieron intoxicaciones". (Médico)$

Los entrevistados mencionaron la necesidad de mayor personal de limpieza para la recolección de desechos y la necesidad de una mayor participación comunitaria en actividades preventivas. Si bien cuentan con un comité vecinal, expresaron ausencia de una participación activa de los pobladores, mencionando inasistencias a las reuniones por dificultades de acceso al cerro o priorización de otras actividades.

"La comunidad debe ir a las charlas informativas, pero la gente es muy ociosa no quiere escuchar y abrimos por gusto, ustedes deberían avisar que va a ver una charla informativa, a ver si la gente va". (Presidente asociación vecinal)

Entre los facilitadores identificados, el personal de salud entrevistado mencionó que en la práctica observan mayor amplitud de los residentes a recibir las pautas que imparten, siempre y cuando el personal de salud sea continuo, insistiendo a pesar de la resistencia de algunos vecinos. Fue mencionado también el trabajo articulado del personal de salud con las asistentas sociales y el personal de escuelas, comisarías y asociaciones vecinales, quienes han recibido capacitaciones en el tema y permiten el acceso a la comunidad durante los brotes. Otra característica facilitadora fue la labor educativa y las actividades realizadas por los promotores de salud.

Finalmente la mayoría de los pobladores expresaron la necesidad de asumir en conjunto la responsabilidad en la prevención del dengue (Tabla 2). Otros entrevistados atribuyeron esta responsabilidad al gobierno local y al personal de salud.

"Yo creo que todos somos responsables, todos debemos estar alertas, porque como le digo, si cae uno caemos todos, entonces hay que estar al tanto, es un riesgo para todos". (Párroco)

\section{Discusión}

Los principales hallazgos de este estudio se pueden resumir brevemente. Entre los primeros tenemos a la percepción de factores modificantes, que incluyen características socioeconómicas, demográficas y/o estructurales, y la información recibida sobre el dengue. Entre las segundas se identificaron, a las barreras y facilitadores para la prevención. Algunas barreras a las actividades preventivas discontinuas son el insuficiente personal de salud y una baja participación comunitaria. Algunos de los facilitadores percibidos fueron la asunción comunitaria de la responsabilidad de la prevención y la realización de actividades por los promotores de salud.

El acceso insuficiente y limitado del agua fue la principal barrera en las medidas de prevención del dengue. Otros estudios también identificaron este factor como importante, observando que las zonas con menor acceso a servicios de agua y desagüe reportaban más casos de dengue ${ }^{24,25}$. La disponibilidad limitada de conexión intra-domiciliaria de agua potable también ha sido asociada con la presencia del vector ${ }^{27,28}$.

Así mismo, se ha identificado al déficit de información como una barrera en la prevención del dengue, hallazgo similar a lo reportado por otros estudios ${ }^{29-31}$, el cual puede provenir de material educativo desactualizado o no contextualizado que conlleva a una baja percepción de la vulnerabilidad hacia la enfermedad por parte de la comunidad ${ }^{27,28}$. El conocimiento insuficiente sobre los criterios 
actuales de diagnóstico, clasificación y tratamiento del dengue encontrado en los médicos generales, se constituye en una barrera más para la prevención del dengue, similar a lo encontrado en la región Lambayeque, en donde el nivel de conocimiento sobre el dengue fue asociado con la oportunidad de la capacitación. Ante estas deficiencias de comunicación en relación al dengue encontrados en los profesionales de salud, es natural comprender que a nivel de la población existan percepciones e información inadecuada sobre el dengue que ameriten ser abordados ${ }^{32-34}$.

Siendo la participación comunitaria un concepto complejo e integral, el cual abarca a distintos actores, diversos estudios asocian la baja participación comunitaria, a diversos entes gubernamentales responsables de la prevención ${ }^{29,30,33}$. Las limitaciones del personal de salud para involucrar a la comunidad y la falta de motivación y compromiso comunitario son algunas de las causas reportadas sobre la escasa participación comunitaria ${ }^{33}$. Del mismo modo se ha reportado una baja percepción del beneficio de las actividades preventivas como resultado de la falta de una acción concertada entre los servicios de salud y la comunidad, sin participación de ésta última y priorizando otros problemas ${ }^{27,34,35}$. De esta manera tanto los factores expresados en este estudio junto con los factores de las otras evidencias sostienen el conjunto de integralidad que engloba la participación comunitaria, pudiendo ser priorizada dese distintos enfoques según el contexto local.

Diversos estudios han empleado el enfoque de ecosalud en el abordaje de contextos de baja participación comunitaria, propiciando acciones intersectoriales en la planificación, ejecución, y evaluación de actividades preventivas y en la gestión del ecosistema para prevenir la transmisión del Aedes aegypti, desarrollando acciones de prevención y control oportunos e integrados ${ }^{35,36}$. Estos enfoques requirieron, al igual que el presente estudio, partir del análisis holístico del problema, para continuar con su priorización y la gestión de sus soluciones, sosteniéndose años después de concluido el proceso ${ }^{36,37}$.

Otras evidencias han identificado como barreras de las acciones preventivas a la sensación de poca autoeficacia, a la percepción deficiente de los beneficios de una potencial acción preventiva y a la insuficiente susceptibilidad percibida ${ }^{38}$. Si bien nuestros hallazgos encontraron que la comunidad reconoce ser vulnerable y susceptible al dengue, también se encontró que el control deficiente de los criaderos a nivel colectivo representaría una de las principales barreras. En este contexto las actividades de prevención basada en la participación comunitaria y con la colaboración de diversos sectores representan una oportunidad para la disminución de los focos de re-infestación en estas comunidades.

En un estudio realizado en Malasia, en relación a la severidad percibida se encontraron dos posibilidades; la primera en la que es posible curarse de la enfermedad y la segunda en la que el desenlace es la muerte ${ }^{38}$. Finalmente, el reporte de un estudio Mexicano, sostiene que el identificar la vulnerabilidad de la comunidad permite identificar necesidades locales particulares que deben ser reforzadas en la labor preventiva promocional además de reportar una asociación significativa entre el incremento de la vulnerabilidad y la prevalencia de los casos reportados ${ }^{26}$.

En el presente estudio, diversos actores identificaron a las charlas educativas como un facilitador para la prevención del dengue, con la participación de instituciones religiosas y educativas; actividad referida previamente por otros estudios ${ }^{30,34}$. En tal sentido, uno de los estudios recomienda que la comunicación se debe centrar en el diálogo, permitiendo que tanto los promotores como los participantes tengan libertad en relación a los temas emergentes durante las charlas, de manera que puedan reconocerlos y discutirlos en el contexto local ${ }^{39}$. Igualmente se ha recomendado que la discusión de la enfermedad no se realice de manera abstracta si no enfocada en el contexto local, en un tiempo y espacio delimitado, y teniendo en cuenta las consideraciones de la comunidad ${ }^{40}$.

Se describe al control de viviendas, uso de abate, familias con buen nivel de información, depósitos tapados como los factores protectores en la prevención del dengue identificados tanto por los pobladores, líderes y personal de salud; similar a lo encontrado en la ciudad de Trujillo ${ }^{41}$.

Consideramos que asumir una responsabilidad compartida en el control del vector representa una potencial oportunidad de unificar conceptos de participación comunitaria, y de conciliar intereses entre usuarios y proveedores de servicios, para lograr una real movilización social.

Una de las limitaciones del presente estudio fue la posibilidad de sesgo de información por la sensación de confrontación durante las entrevistas. A fin de minimizarlo se empleó una estrategia 
de entrevista abierta e imparcial, preguntando directamente sobre las percepciones tanto de los entrevistados como del colectivo o de sus pares, permitiendo obtener las percepciones del entorno del participante.

El presente estudio, sugiere distintos grados de reconocimiento hacia la susceptibilidad y severidad al dengue manifestado principalmente por el insuficiente acceso: a servicios básicos de saneamiento, las actividades preventivas discontinuas y el escaso personal de salud, así como, la escasa participación comunitaria. No obstante, las acciones de los promotores de salud, la autopercepción de vulnerabilidad y de responsabilidad hacia la transmisión del dengue podría facilitar el abordaje de la superación de algunas de las barreras mencionadas.

\section{Contribución}

Gloria Carmona, contribuyó en el planteamiento, ejecución, análisis de los hallazgos y redacción del manuscrito inicial y final de esta investigación. Luís Donaires, contribuyó activamente en el análisis y discusión de los hallazgos, además de su participación crítica en la revisión y la aprobación final del manuscrito.

\section{Referencias}

1. Gubler DJ. Epidemic dengue/dengue hemorrhagic fever as a public health, social and economic problem in the 21st century. Trends Microbiol. 2002; 10(2):100-3.

2. UNDP, World Bank, WHO Special Programme for Research \& Training in Tropical Diseases. Meeting report. Recommendations: Scientific working group on dengue [Internet]. Geneva, Switzerlandt; 2000 Apr [cited 2014 Apr 30]:12. Available from: http://www.who.int/tdr/publications/documents/dengue-swg.pdf?ua=1

3. San Martín JL, Brathwaite O, Zambrano B, Dayan GH, Solórzano JO, Bouckenooghe $A$, et al. The epidemiology of dengue in the americas over the last three decades: a worrisome reality. Am J Trop Med Hyg. 2010; 82(1):128-35.

4. Lourdes Sulca Cachay SA. Reappearance of Aedes aegypti (Diptera: Culicidae) in Lima, Peru. Mem Inst Oswaldo Cruz. 2001; 96(5):657-8.

5. Oficina General de Epidemiología. Extensión del dengue en el Perú [Internet]. Lima; 2005 [acesso 2015 Ene 4]. Disponible en: http://www.saludarequipa.gob.pe/ epidemiologia/bole_epi/dge2005/Bolet_15.pdf

6. Oficina General de Epidemiología. Aspectos epidemiológicos del dengue: perspectiva del control vectorial. Ministerio de salud. Lima, Perú. Report. 2005; 14(18):8-12.

7. Soto C. Informe final brote dengue clásico: la fragata y Zarate, San Juan de Lurigancho. Disa IV Lima este, abril-mayo 2007. 
8. Ministerio de Salud (PE). Escenarios epidemiológicos de riesgos por dengue [Internet]. Dirección de Salud II Lima Sur [acesso 2014 Nov 15]. Disponible en: http://disalimasur. gob.pe/arch_documentos/archivos/1373670997.pdf

9. Unidad de epidemiologia. Indice de infestacion aedica micro redes [Internet]. Red de Salud Túpac Amaru [acesso 2015 Ene 22]. Disponible en: http://www.minsarsta.gob.pe/ pagweb/epidemiologia/sala\% 20situacional\% 202013/SALA\% 20DENGUE\% 20SE\% 20142013.pdf

10. Dirección de Salud II Lima Sur. Primer brote de dengue en Lima Sur [Internet] [acesso 2015 feb 21]. Disponible en: http://bvs.minsa.gob.pe/local/minsa/2691.pdf

11. Decreto Supremo que aprueba el Plan Nacional Multisectorial e Intergubernamental de Prevención y Control de Dengue en el Perú [Internet]. Lima: Ministerio de Salud; 2013 [acesso 2015 Jun 14]. Disponible en: ftp://ftp2.minsa.gob.pe/normaslegales/2013/ DS007_2013_SA_b_EP.pdf

12. Ballenger-Browning KK, Elder JP. Multi-modal Aedes aegypti mosquito reduction interventions and dengue fever prevention. Trop Med Int Health. 2009;14(12):1542-51.

13. Concepción Rojas M, Cifuentes E, Rodríguez Jiménez R, Valdés Ramos I. Indicadores del ecosistema para la prevención y control del dengue en el municipio de Cotorro (Cuba). Rev Hig Sanid Ambient. 2006; (6):138-44.

14. Hoyos Rivera A. Intervención comunitaria en el dengue como una necesidad social. Rev Cubana Salud Publica. 2011; 37(4):500-9.

15. Wai KT, Htun PT, Oo T, Myint H, Lin Z, Kroeger A, et al. Community-centred eco-biosocial approach to control dengue vectors: an intervention study from Myanmar. Pathog Glob Health. 2012; 106(8):461-8.

16. Gubler DJ, Clark GG. Community involvement in the control of Aedes aegypti. Acta Trop. 1996;61(2):169-79.

17. Sanchez L, Perez D, Pérez T, Sosa T, Cruz G, Kouri G, et al. Intersectoral coordination in Aedes aegypti control. A pilot project in Havana City, Cuba. Trop Med Int Health. 2005; 10(1):82-91.

18. Schaffner F, Bellini R, Petric D, Scholte E-J, Zeller H, Marrama Rakotoarivony L. Development of guidelines for the surveillance of invasive mosquitoes in Europe. Parasit Vectors. 2013; 18(6):209.

19. Harrison JA, Mullen PD, Green LW. A meta-analysis of studies of the Health Belief Model with adults. Health Educ Res. 1992; 7(1):107-16.

20. Organización Panamericana de la Salud. Plan detallado de acción para la próxima generación: prevención y control del dengue [Internet]. Programa de enfermedades transmisibles. División de Prevención y Control de Enfermedades [acesso 2015 mar 5]. Disponible en: http://www.bvsde.paho.org/bvsair/e/repindex/repi78/pagina/text/e/ redengue/plan/plan.pdf

21. Díaz C. Preventing Dengue at the local level in Havana city. In: Charron DF, editor. Ecohealth research in practice [Internet]. Springer New York; 2012. p. 163-71 [cited 2014 Apr 1]. (Insight and Innovation in International Development). Available from: http://link. springer.com/chapter/10.1007/978-1-4614-0517-7_15

22. Bernard H. Social Research Methods. Qualitative and quantitative approaches. 2a ed. Florida: Sage Publications; 2013.

23. Steckler A, McLeroy KR, Holtzman D. Godfrey H. Hochbaum (1916-1999): from social psychology to health behavior and health education. Am J Public Health. 2010;100(10):1864. doi:10.2105/AJPH.2009.189118. 
24. Fernández de Córdova G. Nuevos patrones de segregación socio-espacial en Lima y Callao 1990-2007 [Internet]. Lima: Pontificia Universidad Católica del Perú, Departamento de Arquitectura; 2012 [cited 2015 Jan 7]. Disponible en: http://repositorio.pucp.edu.pe/ index//handle/123456789/35703

25. Tana S, Abeyewickreme W, Arunachalam N, Espino F, Kittayapong P, Wai KT, et al. Eco-Bio-Social research on dengue in Asia: general principles and a case study from Indonesia. In: Charron DF, editor. Ecohealth research in practice [Internet]. York: Springer; 2012. p. 173-84 [cited 2014 Apr 1]. Available from: http://link.springer.com/ chapter/10.1007/978-1-4614-0517-7_16

26. Tong A, Sainsbury P, Craig J. Consolidated criteria for reporting qualitative research (COREQ): a 32-item checklist for interviews and focus groups. Int J Qual Health Care. 2007; 19(6):349-57.

27. Escobar-Mesa J, Gómez-Dantés H. Determinantes de la transmisión de dengue en Veracruz: un abordaje ecológico para su control. Salud Publica Mex. 2003; 45(1):43-53.

28. Súarez-Ognio L, Arrasco J, Casapía M, Sihuincha M, Avila J, Soto G, et al. Factores asociados a dengue grave durante la epidemia de dengue en la ciudad de Iquitos, 20102011. Rev Peru Epidemiol Online [Internet]. 2011 [cited 2014 Nov 21]; 15(1):17-23. Available from: http://rpe.epiredperu.net/rpe_ediciones/2011_V15_N01/3AO_Vol15_ No1_2011_Dengue_lquitos.pdf

29. Wong LP, AbuBakar $S$. Health beliefs and practices related to dengue fever: a focus group study. PLoS Negl Trop Dis. 2013; 7(7):e2310.

30. Pérez-Guerra CL, Zielinski-Gutierrez E, Clark GG. Community beliefs and practices about dengue in Puerto Rico. Rev Panam Salud Publica. 2009; 25(3):218-26.

31. Phuanukoonnon S, Brough M, Bryan JH. Folk knowledge about dengue mosquitoes and contributions of health belief model in dengue control promotion in Northeast Thailand. Acta Trop. 2006; 99(1):6-14.

32. Paico-Romero C, Polo-Capuñay A, Díaz-Exebio G, Díaz-Vélez C. Validación y desarrollo del instrumento: nivel de conocimientos en diagnóstico y tratamiento sobre dengue en médicos de la región Lambayeque. Acta Médica Peru. 2012; 29(3):143-7.

33. Romaní $M$, Gil AB, Ursula EC, Boelaert M, der Stuyft PV. Participación comunitaria en la prevención del dengue: un abordaje desde la perspectiva de los diferentes actores sociales. Salud Publica Mex. 2006; 48(1):39-44.

34. Schweigmann N, Rizzotti A, Burroni N, Castiglia G, Gribaudo F, Marcos E, et al. Information, knowledge, and perception of dengue risk in Argentina: two intervention experiments to generate local control strategies. Cad Saude Publica. 2009; (25):S137-48.

35. Smith V. A qualitative analysis: using the Health Belief Model to explain dengue fever in two communities in Trinidad and Tobago. J Dep Behav Sci. 2012; 2(1):90-101.

36. Díaz C, Torres $Y$, Cruz AM, Álvarez ÁM, Piquero ME, Valero A, et al. An intersector participatory strategy in Cuba using an ecosystem approach to prevent dengue transmission at the local level. Cad Saude Publica. 2009; (25):S59-70.

37. Melián MG, Alonso MÁM, Pantoja CD, Rojas MC, Ramos IV. El componente ambiental de la vigilancia integrada para el control y la prevención del dengue. Rev Cuba Hig Epidemiol [Internet]. 2007 [cited 2014 May 2]; 45(1). Available from: http://www. redalyc.org/resumen.oa?id=223219928007

38. Wong LP, AbuBakar $\mathrm{S}$. Health Beliefs and practices related to dengue fever: a focus group study. PLoS Negl Trop Dis. 2013; 7(7):e2310.

39. Silva LB, Soares SM, Aquino AL. Seasonal communication about dengue fever in educational groups in primary healthcare. Rev Saude Publica. 2011; 45(6):1160-7. 
40. Soares de Araújo I. Contextos, mediações e produção de sentidos: uma abordagem conceitual e metodológica em comunicação e saúde. Rev Eletron Comun Inf Inov Saude. 2009; 3(3):42-50.

41. Alfaro M, Luna M, Muñoz M, Novoa Y, Castillo N, Benites S, et al. Factores fundamentales de exposición al dengue según escenarios geoepidemiológicos en Trujillo Metropolitano. Abril-Mayo 2001. Rev Peru Epidemiol Online. 2002; (10):1-8.

Carmona G, Donaires LF. Percepções comunitárias na prevenção da dengue nos assentamentos humanos. Lima-Peru, 2015. Interface (Botucatu). 2016; 20(59):839-52.

Com o objetivo de descrever as percepções comunitárias a respeito da prevenção da dengue, realizou-se um estudo qualitativo nas áreas afetadas de Lima. Foram utilizadas entrevistas sob o modelo de crenças em saúde. Os entrevistados mencionaram diversos fatores de saneamento e condutas que aumentariam sua exposição à doença. Também enumeraram aspectos laborais, familiares e econômicos que seriam afetados pelo adoecimento. Os entrevistados assumiram parte da responsabilidade na prevenção, mas destacaram a importância do trabalho dos promotores de saúde, líderes comunitários e meios de comunicação. Dentre as medidas preventivas enumeradas, foram citadas algumas que não tinham evidência de efetividade. Os principais obstáculos citados foram: quantidade insuficiente de profissionais de saúde, baixa participação comunitária, desorganização das atividades preventivas e descontinuidade das ações após a resolução dos surtos. A discussão dos resultados encontrados promove a oportunidade de refletir sobre o trabalho preventivo realizado para o combate à dengue.

Palavras-chave: Dengue. Percepção social. Ação intersetorial. Assentamentos humanos.

Submetido em 15/05/15. Aprovado em 22/02/16. 